\title{
ESTUDO DA SUSCETIBILIDADE DA BIOMPHALARIA GLABRATA DE OURINHOS (SP), Ȧ INFECÇÃO PELO SCHISTOSOMA MANSONI DE BELO HORIZONTE (MG), E DE SÃO JOSE DOS CAMPOS (SP), *
}

\begin{tabular}{l|l|} 
RSPU-B/181 \\
\hline
\end{tabular}

Magalhães, L. A. \& Dias, L. C. de S. - Estudo da suscetibilidade da Biomphalaria glabrata de Ourinhos (SP), à infecção pelo Schistosoma mansoni de Belo Horizonte (MG), e de São José dos Campos (SP). Rev. Saúde públ., S. Paulo, 7: 295-7, 1973.

Resumo: Foi estudada a suscetibilidade da Biomphalaria glabrata de um foco de Schistosoma mansoni no município de Ourinhos, (SP, Brasil). Concluiu-se pela alta capacidade desses moluscos à infecção pelas cepas de .S. mansoni de Belo Horizonte, Minas Gerais e de São José dos Campos, São Paulo.

URItermos: Biomphalaria glabrata (Suscetibilidade)*; Schistosoma mansoni *; Esquistossomose *.

Tratamos de estudar a suscetibilidade de exemplares de Biomphalaria glabrata de Ourinhos, SP, à infecção por miracídios de Schistosoma mansoni das linhagens de Belo Horizonte, MG e de São José dos Campos, SP.

Focos de esquistossomíase mansônica em criadouros de $B$. glabrata têm sido assinalados em Ourinhos. $\operatorname{REY}_{7}$ (1952), encontrou $3,2 \%$ de $B$. glabrata naturalmente infectadas nesta cidade. Em 1960, PIZA \& RAmos 6 referiram um índice de infecção de $0,08 \%$ para moluscos da mesma região. Focos de $S$. mansoni em Ourinhos continuam em atividade, apre- sentando sempre baixos índices de infecção natural.

\section{MATERIAL E METODOS}

Utilizamos na experiência exemplares de $B$. glabrata nascidos em laboratório, provenientes de espécimes capturados em foco de S. mansoni, no município de Ourinhos. Os caramujos escolhidos para a experiência mediam de 2 a $12 \mathrm{~mm}$ de diâmetro máximo. Após separados em dois lotes de 20 moluscos, os exemplares foram colocados individualmente em pequenas placas de Petri que continham água e 10 miracídios de S. man soni. As placas contendo moluscos e

* Do Instituto de Biologia da Universidade Estadual de Campinas. - Caixa Postal 1170 - Campinas, S.P. - Brasil. 
MAGALHAES, L. A. \& DIAS, L. C. de S. - Estudo da suscetibilidade da Biomphalaria glabrata de Ourinhos (SP), à infecção pelo Schistosoma mansoni de Belo Horizonte (MG), e de São José dos Campos (SP). Rev. Saúde públ., S. Paulo, 7:295-7, 1973.

miracídios eram expostas à ação do calor emanado por lâmpadas de $60 \mathrm{~W}$, durante duas horas. No primeiro lote utilizamos miracídios de $S$. mansoni da linhagem de Belo Horizonte ( $\mathrm{BH}$ ) e no segundo lote utilizamos miracídios da linhagem de São José dos Campos (SJ).

A contar 30 dias a partir da data da infecção, os moluscos eram diariamente examinados após exposição por duas horas, à ação de lâmpadas elétricas.

R E S U L T A D O S

Todos os caramujos utilizados na experiência mostraram-se infectados por $S$. mansoni. Nos dois lotes, as primeiras cercárias de $S$. mansoni foram encontradas no $42 .^{\circ}$ dia, após a data da infecção.

\section{Discussáo E Conclusao}

É referida a resistência à infecção cruzada, entre as linhagens de $S$. mansoni mantidas na natureza, por $B$. glabrata e por B. tenagophila.

Paraense \& CorrêA 5 observaram que moluscos B. tenagophila do Vale do Rio Paraíba, SP, mostraram-se facilmente infectados por miracídios de $S$. mansoni da mesma região, porém resistentes à infecção por miracídios oriundos da linhagem mineira de $S$. mansoni, mantida no campo ou em laboratório através da infecção por $B$. glabrata. Verificaram também que $B$. glabrata de Minas Gerais era resistente à infceção pela cepa paulista de $S$. mansoni.

Estudando a suscetibilidade à infecção pelo S. mansoni dos planorbídios de Campinas, verificamos que esses moluscos se comportam, sob este aspecto, de maneira similar aos seus co-específicos do Vale do Rio Paraíba ${ }^{3}$.
Barbosa \& Barreto ${ }^{1}$ assinalaram que a diferença de suscetibilidade apresentada à infecção pelo $S$. mansoni por duas cepas brasileiras de $B$. glabrata correriam por conta dos moluscos e não pelo trematódeo infectante. Mais tarde Paraense \& Corrếa 4 referiram que a variação na suscetibilidade de populações de $B$. glabrata à diferentes linhagens de S. mansoni, devia ser considerada como um aspecto de variação intra-específica, relacionada com genotipo da população de caramujos.

Em trabalho anterior 2 sugerimos que a diferença de suscetibilidade à infecção pelo S. mansoni apresentada pelos planorbídeos, além de estar relacionada com o comportamento do miracídio infectante, eventualmente dependeria de variaçōes inter-específicas apresentadas pelos moluscos.

No presente trabalho verificamos que a $B$. glabrata de Ourinhos apresentou-se, ao contrário de sua co-específica mineira igualmente suscetivel à infecção pelo $S$. mansoni de São José dos Campos e de Horizonte.

Em vista dos resultados obtidos até o presente trabalho, achamos que a questão de suscetibilidade dos planorbídeos à infecção pelo $S$. mansoni esteja na dependência do comportamento das linhagens do verme e do caramujo que apresentariam variações, sob este aspecto, intra e inter-específicas.

O fato de ser a $B$. glabrata de Ourinhos altamente suscetível à infecção pelas duas linhagens referidas de $S$. mansoni, facilitaria, na região habitada pela população estudada, o intercâmbio genético entre as linhagens mineira e paulista do trematódeo. 
MAGALHÃES, L. A. \& DIAS, L. C. de S. - Estudo da suscetibilidade da Biomphalaria glabrata de Ourinhos (SP), à infecção pelo Schistosoma mansoni de Belo Horizonte (MG), e de Sáo José dos Campos (SP). Rev. Saúde públ., S. Paulo, 7:295-7, 1973.

\section{AGRADECIMENTO}

Ao engenheiro Luís Takaku, da Campanha de Combate à Esquistossomose, pelo envio dos moluscos de Ourinhos, cujos descendentes foram utilizados neste trabalho.

RSPU-B/181

MAGALHÃES, L. A. \& Dias, L. C. de S. - [Susceptibility of Biomphalaria glabrata from Ourinhos, SP to schistosomiasis infection by Schistosoma mansoni strain from Belo Horizonte, $M G$ and S. José dos Campos, SP (Brazil.)] Rev. Saúde públ., S. Paulo, 7: 295-7, 1973.

Summary: The susceptibility of Biomphalaria glabrata from Ourinhos, São Paulo, to strains of Schistosoma mansoni from Belo Horizonte, Minas Gerais and S. José dos Campos, S. Paulo, was studied. It was conclude that the molluscs has a high susceptibility to both strains of Schistosoma mansoni.

UNITERMS: Biomphalaria glabrata, susceptibility* Schistosoma mansoni*; Schistosomiasis *.

\section{REFERENCIAS BIBLIOGRAFICAS}

1 - BARBOSA, F. S. \& BARRETO, A. C. Differences in susceptibility of Brazilian strains of Australorbis glabratus to Schistosoma mansoni. Exper. Parasit. 9:137-40, 1960.

2 - Magalhaes, L. A. - Moluscos planorbideos do Distrito Federal, Brasília. Campinas, 1967. [Tese de Doutoramento - Universidade Estadual de Campinas]

3 - Magalhăes, L. A. \& CaRvalho, J. F. - Estudo dos dados obtidos de uma população de Biomphalaria glabrata de Belo Horizonte infectada por Schistosoma mansoni da mesma cidade, e de uma população de $B$. tenagophila de Campinas infectada por $S$. mansoni de São José dos Campos. Rev. Soc. bras. Med. trop. 3:195-6, 1969 .
4 - PARAENSE, W. L. \& CORRÊA, L. R. Variation in susceptibility of populations of Australorbis glabratus to a strain of Schistosoma mansoni. Rev. Inst. Med. trop. São Paulo, 5:15-22, 1963.

5 - PARAENSE, W. L. \& CORREA, L. R. Susceptibility of Australorbis tenagophilus to infection with Schistosoma mansoni. Rev. Inst. Med. trop S. Paulo, 523-9, 1963.

6 - PIZA, J. T. \& RAMOS, S. A. - Os focos autóctones de esquistossomose no Estado de Sáo Paulo. Arq. Hig. S. Paulo, 25:261-71, 1960.

7 - REY, L. - Primeiro encontro de planorbídeos naturalmente infectados por furcocercárias de $S$. mansoni no planalto paulista. Rev. clin. S. Paulo,2, $28(5-6): 57-64,1952$. 